Karlsruhe Institute of Technology

\title{
Analysis of EM loads on DEMO WCLL Breeding Blanket during VDE-up
}

\author{
Ivan A. Maione ${ }^{a}$, Massimo Roccella ${ }^{b}$, Flavio Luccab ${ }^{b}$ Anna Marin ${ }^{b}$, Claudio Bertolinib,
} Fabio Villone ${ }^{c}$, Alessandro Del Nevo ${ }^{d}$

\author{
a Karlsruhe Institute of Technology (KIT), Hermann-von-Helmholtz-Platz 1, 76344 Eggenstein-Leopoldshafen, Germany \\ bLT Calcoli srl, Via Bergamo 60, 23807 Merate (LC), Italy \\ 'CREATE, DIEl, Università di Cassino e del Lazio Meridionale, Cassino (FR), Italy
}

'ENEA FSN-ING-PAN C.R. Brasimone, 40032 Camugnano (Bo), Italy

This work presents the development of a DEMO EM model based on the WCLL design able to evaluate EM loads during normal and off-normal operations. The model has the capability to use periodic boundary conditions and elements supporting non-linear magnetic properties. Eddy currents and related EM loads are calculated using ANSYS Emag and considering a VDE-up provided by CarMaONL code with a 74 ms current quench time. Both the poloidal and toroidal field variations due to the plasma movement and to the current quench are implemented. The obtained results represent an important input for the structural assessment of the BB segments as well as for the definition of the attachment system with the VV.

\section{- Electromagnetic FEM model}

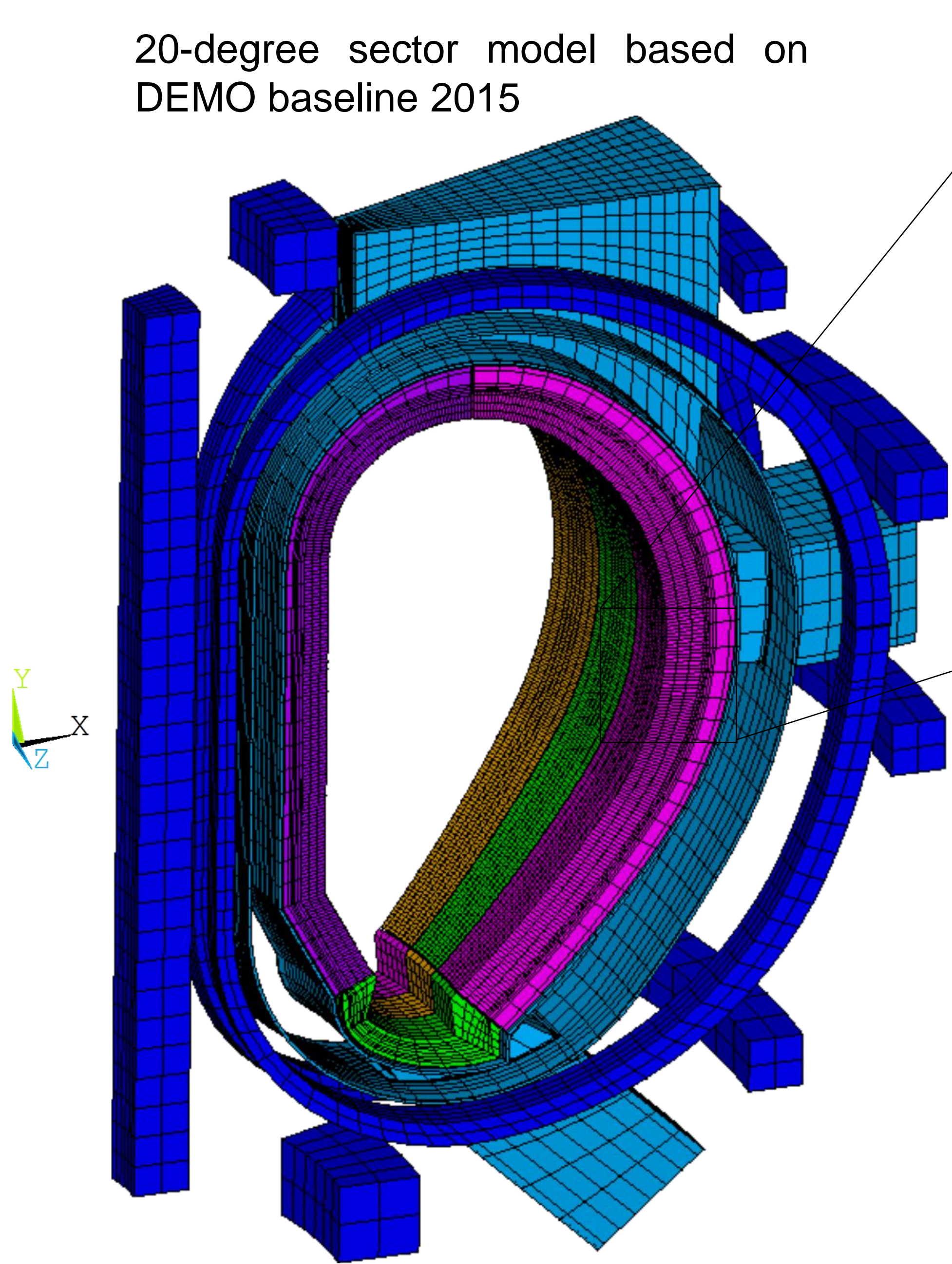

Averaged materials and EM properties have been used for the conductive components defined in the WCLL FEM model.

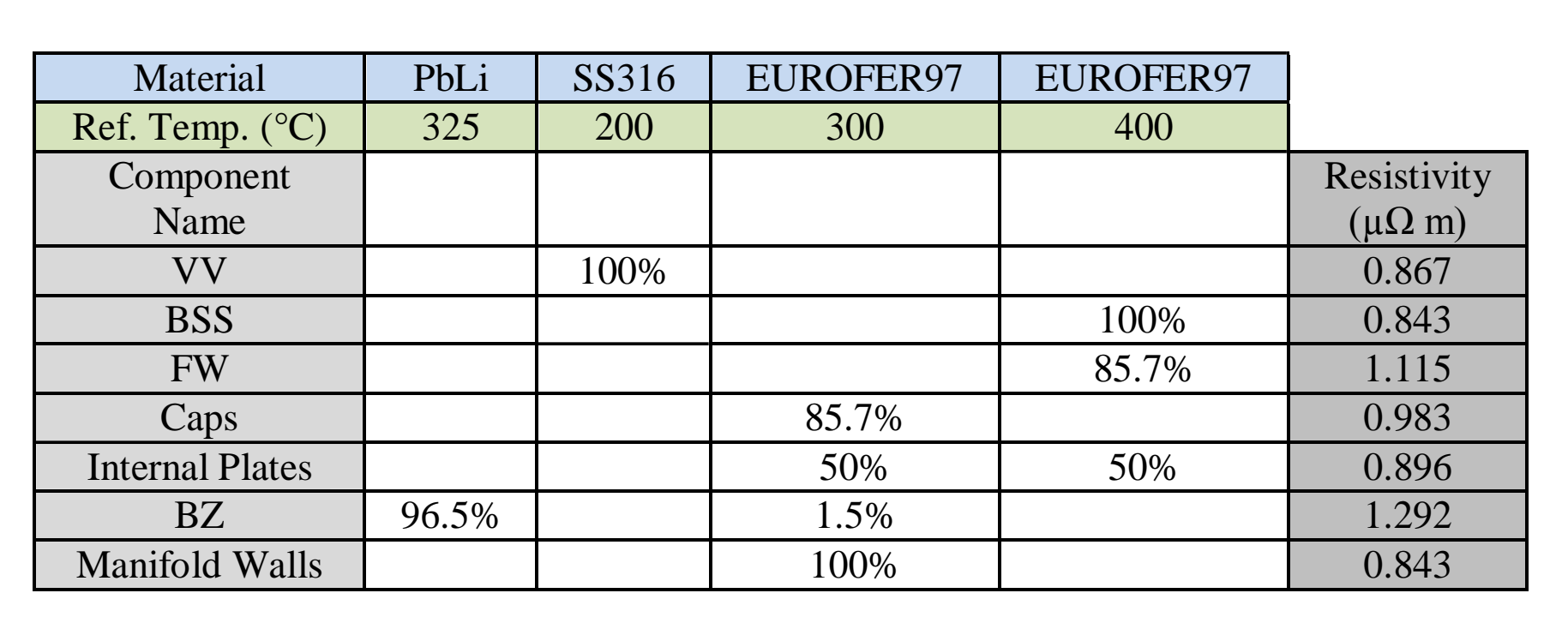

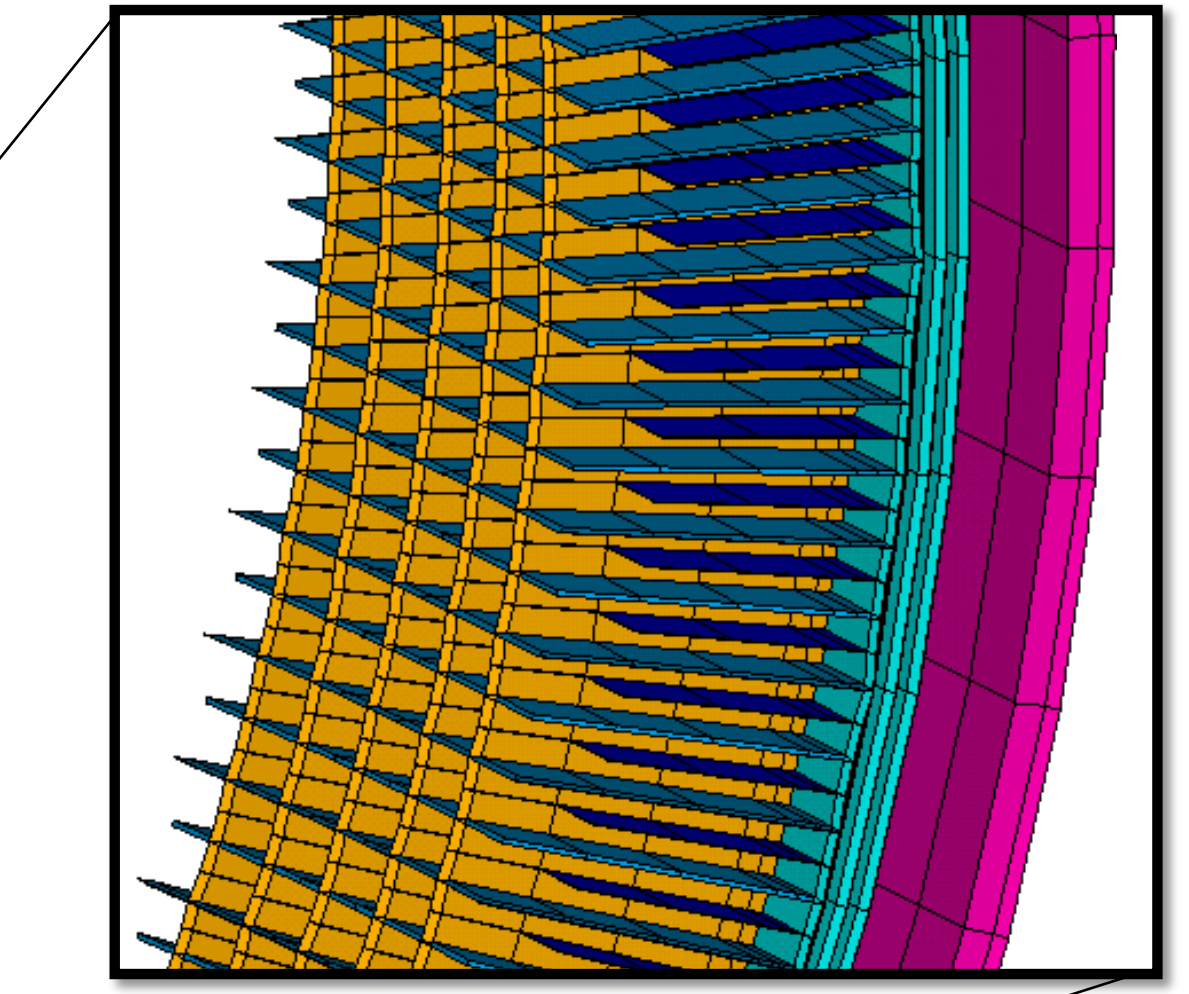

The internal structure of the WCLL design consisting in Pol-rad, Tor-rad and baffle plates has been implemented.

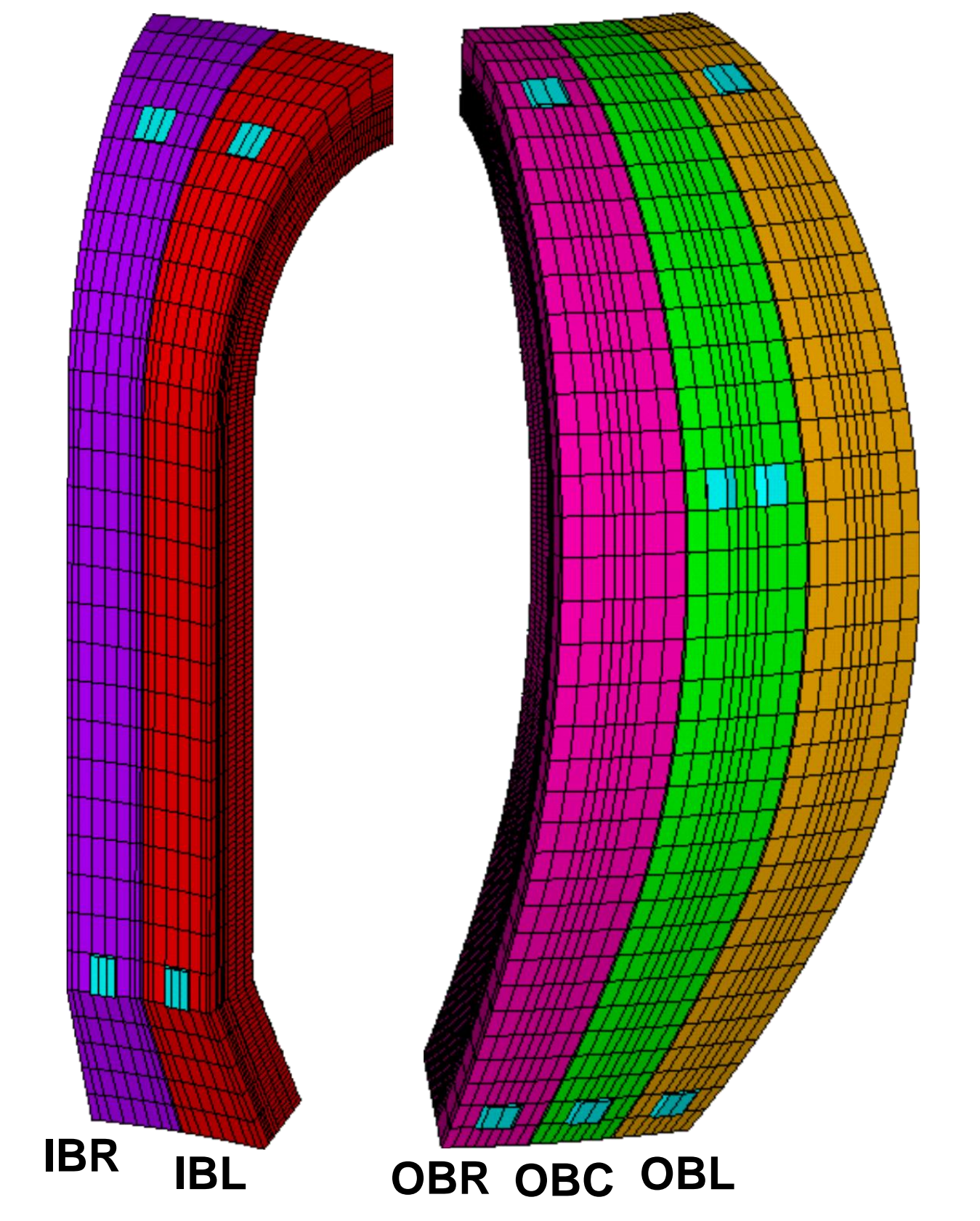

Electrical connections between BBs and $V V$ have been implemented at the top and bottom of the blanket structure following the design of the attachment system.

\section{- Loads and boundary conditions}

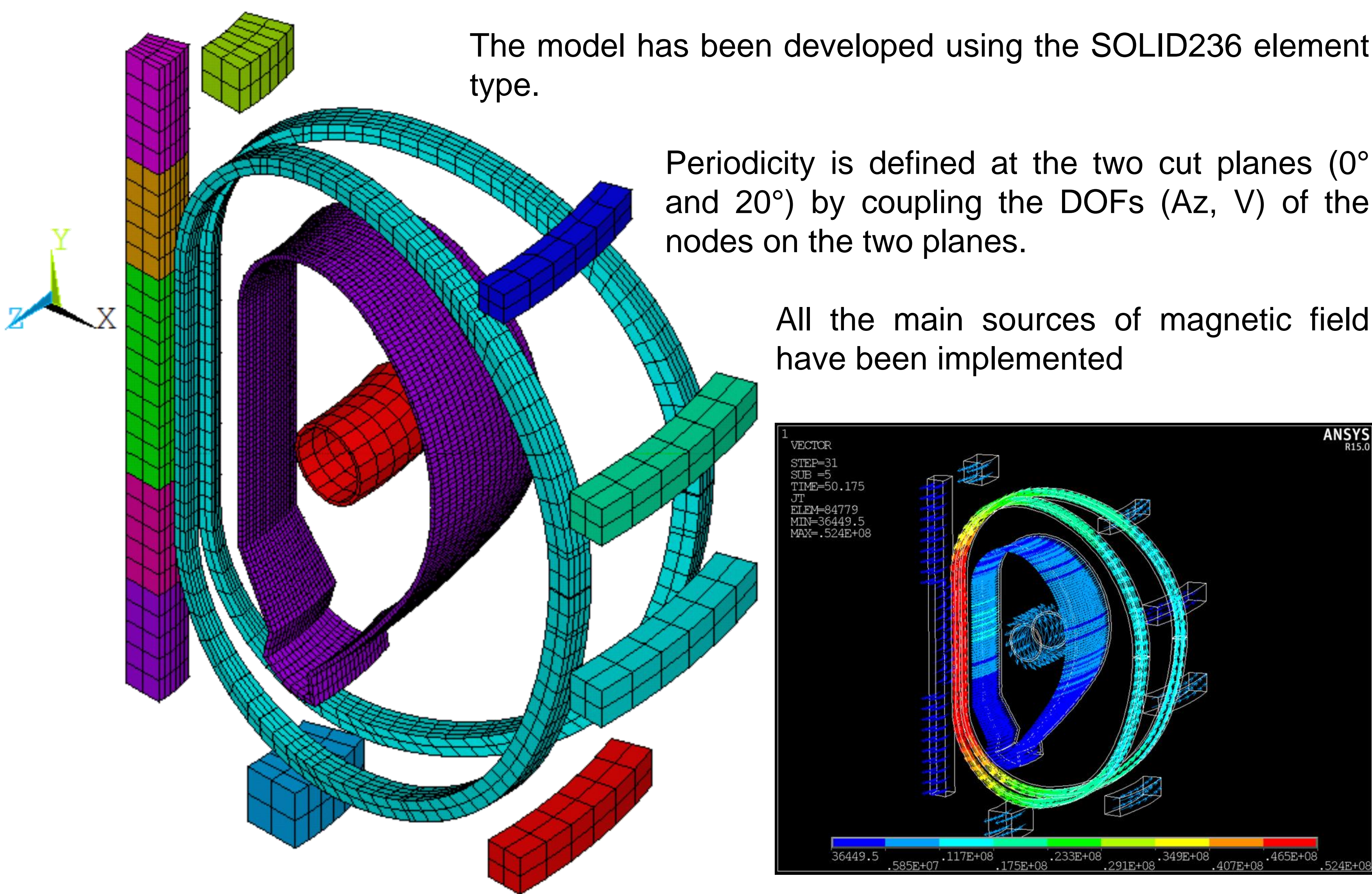

Poloidal coils' currents and plasma input (both poloidal and toroidal contribution) have been calculated by CarMa0NL code and provided for a VDE-up with a $74 \mathrm{~ms}$ current quench time.
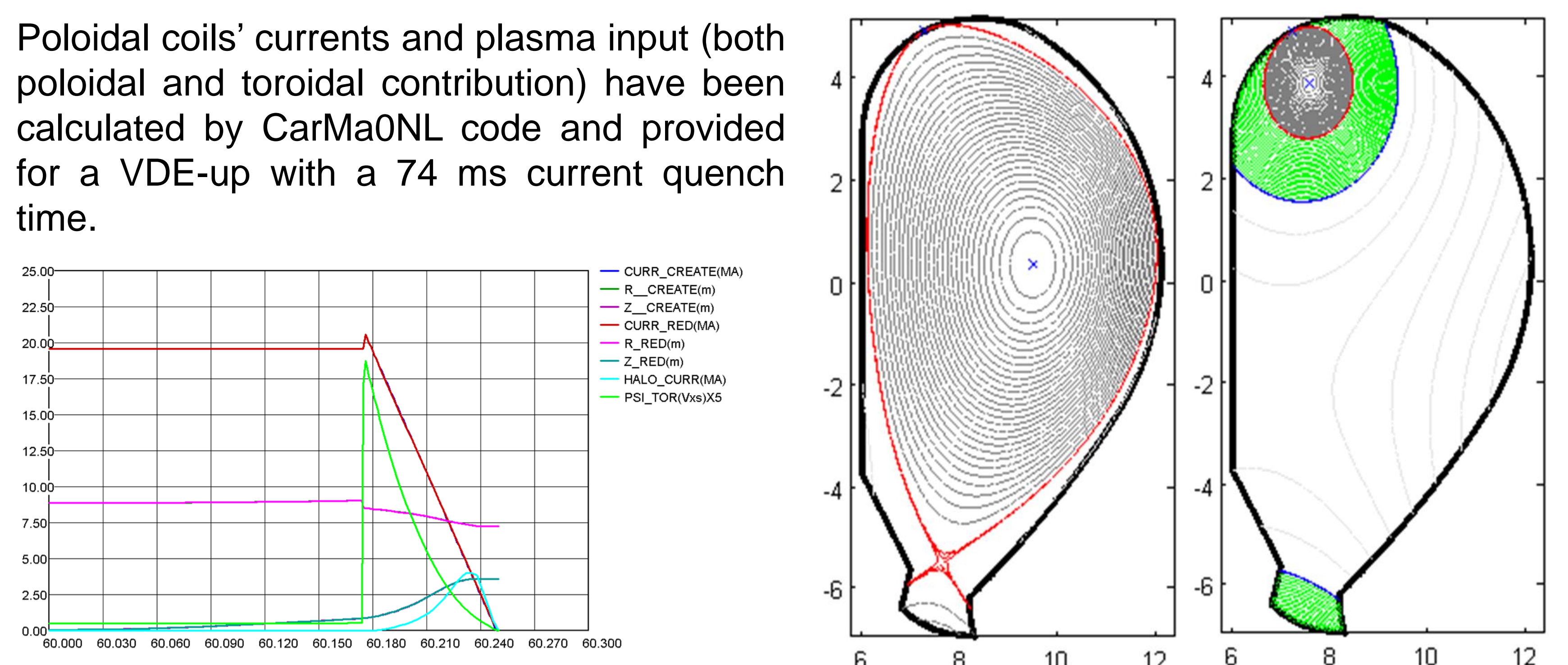

Results

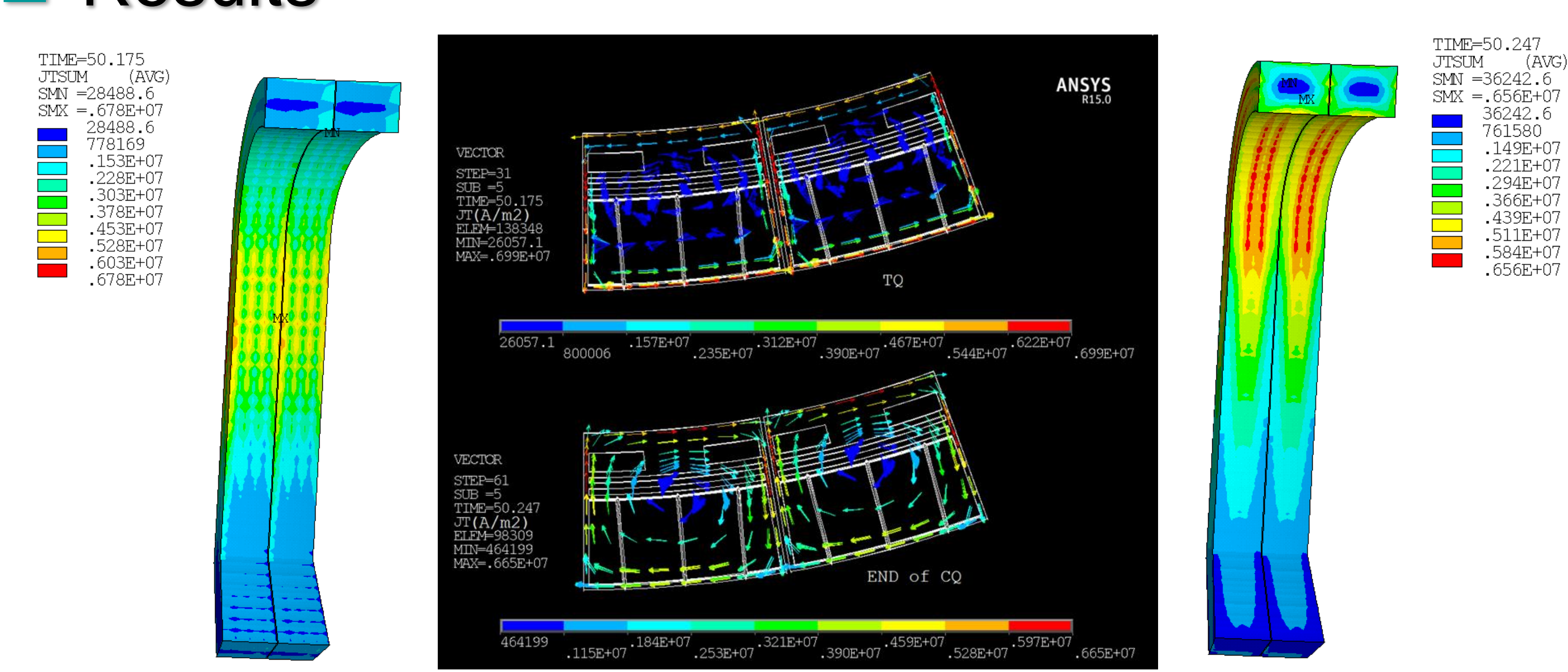

$\mathrm{IB}$ and $\mathrm{OB}$ eddy current distribution at $\mathrm{TQ}$ peak and at the end of the $\mathrm{CQ}$
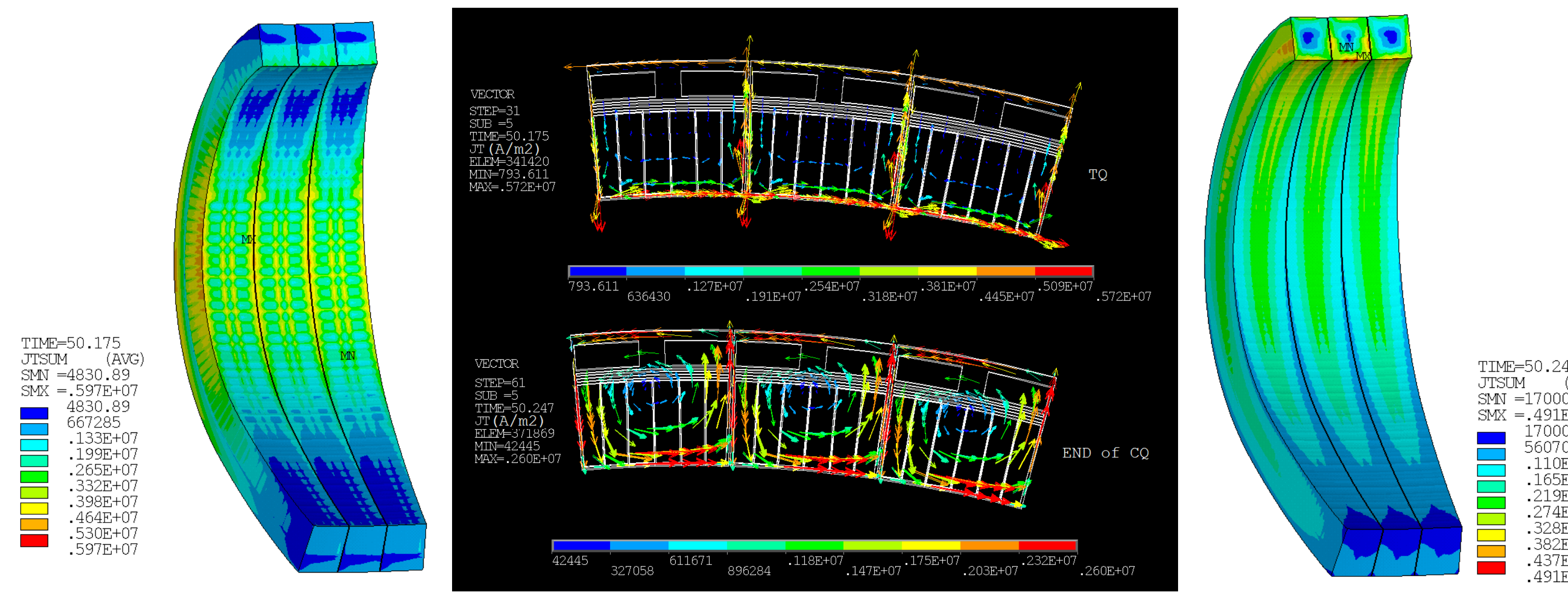

Time behaviour of the IB and OB Lorentz force and moment during the VDE-UP in the local coordinate systems
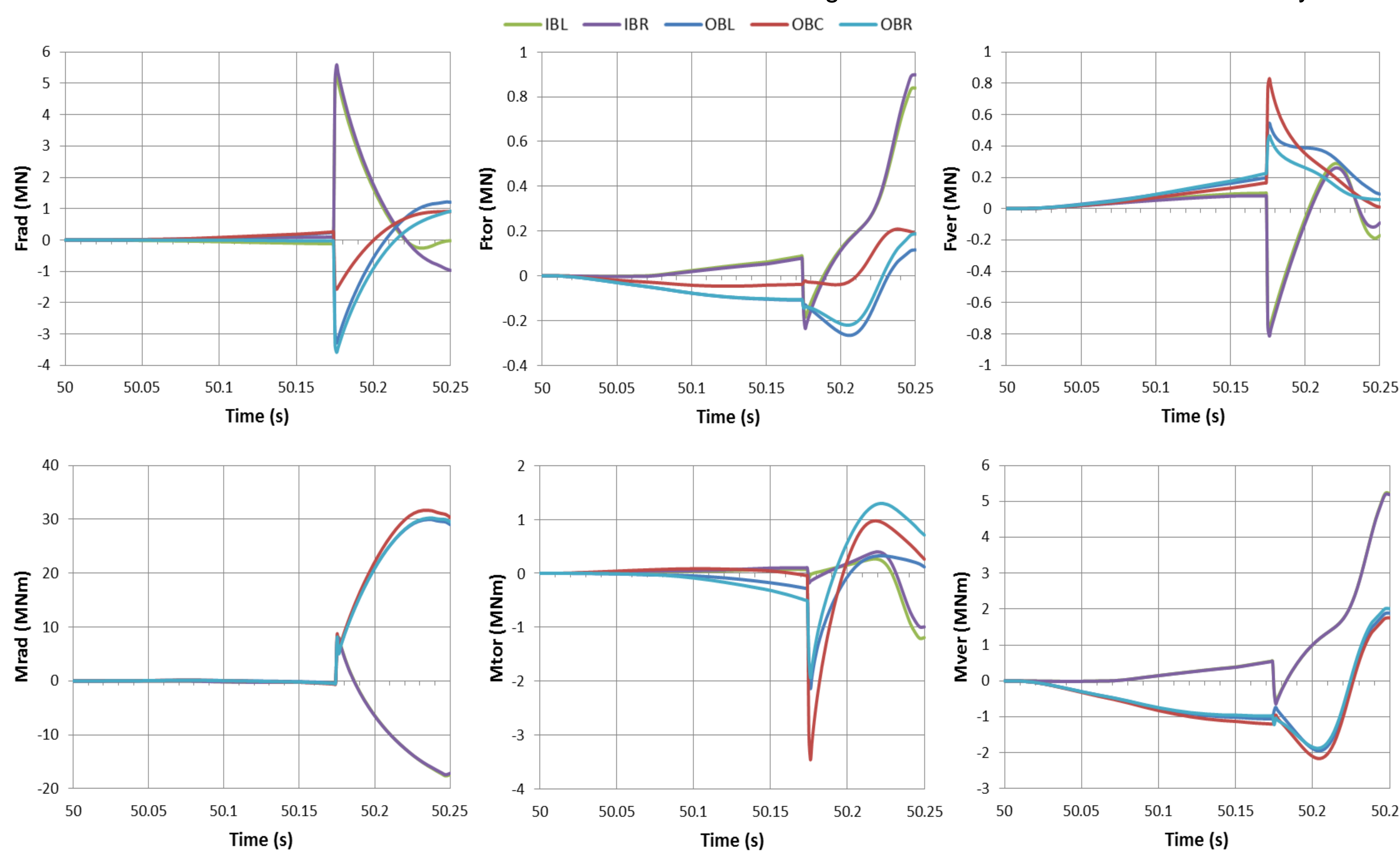